\title{
Originales
}

\section{Intubación traqueal guiada por fibrobroncoscopio en pacientes con vía aérea difícil. Factores predictores del resultado}

\author{
M. BOTANA, A. FERNÁNDEZ-VILLAR, V. LEIRO, C. REPRESAS, A. MÉNDEZ \\ Y L. PIÑEIRO
}

Unidad de Técnicas Broncopleurales. Servicio de Neumología. Hospital Xeral de Vigo. Complexo Hospitalario Universitario de Vigo (CHUVI). Vigo. Pontevedra. España.

Introducción y objetivo. La intubación traqueal (IT) guiada por fibrobroncoscopio (FB) es una de las técnicas esenciales en el abordaje de la vía aérea difícil (VAD). Hay pocos trabajos publicados sobre las causas del fallo de la IT con este procedimiento. El objetivo del presente estudio es analizar cuáles son los factores que podrían predecir el fallo de la IT con FB.

Material y métodos. Estudio observacional retrospectivo en el que se incluyeron las últimas 122 IT consecutivas guiadas por FB (enero de 2000 a abril de 2008) realizadas por nuestro grupo. Se llevó a cabo un análisis multivariable de los factores que podrían influir en el resultado: causa de VAD, vía de IT, tipo de tubo endotraqueal, indicación programada o urgente del procedimiento, grado de sedación y experiencia del broncoscopista.

Resultados. Las IT en individuos que se sometieron a intervenciones quirúrgicas representaron el $92,6 \%$ del total. Las indicaciones más frecuentes de la IT por FB fueron: limitación del movimiento del cuello (60 casos), estenosis de la vía aérea (24), aumento de tejidos blandos (13), apertura oral limitada (9), compresión de la vía aérea (6) y parálisis de cuerdas vocales (6). En $10(8,2 \%)$ casos la IT por FB no fue posible. Las variables que mejor predijeron el fracaso de la IT en el análisis multivariable fueron la sedación profunda/anes-

Correspondencia: Dra. M. Botana Rial

Unidad de Técnicas Broncopleurales. Servicio de Neumología. Hospital Xeral de Vigo. CHUVI.

Pizarro, 22. 36204 Vigo. Pontevedra. España.

Correo electrónico: maria.isabel.botana.rial@sergas.es

Manuscrito aceptado el 17-11-2008. tesia general (odds ratio [OR] = 12,2; intervalo de confianza [IC] del $95 \%, 1,8-84 ; p=0,01)$ y la escasa experiencia del broncoscopista (OR $=25,3$; IC del $95 \%, 3,5-181,8 ; p=0,001$ ).

Conclusiones. En más del $90 \%$ de los casos con VAD, la IT con FB realizada por broncoscopistas es exitosa. La habilidad y la experiencia del broncoscopista son uno de los principales determinantes del éxito del procedimiento. La sedación profunda puede condicionar el fallo de la IT por FB.

PALABRAS CLAVE: Broncoscopia. Vía aérea difícil. Intubación traqueal.

\section{TRACHEAL INTUBATION GUIDED BY FIBROBRONCHOSCOPY IN PATIENTS WITH DIFFICULT AIRWAY. PREDICTIVE FACTORS OF THE OUTCOME}

Introduction and objective. Tracheal intubation (TI) guided by fibrobronchoscopy (FB) is one of the essential techniques in the approach to the difficult airway (DAW). Few works have been published on the possible causes of TI failure with this procedure. This study aims to discover which factors could predict TI failure with FB.

Material and methods. An observational and retrospective study in which the last 122 consecutive Tls guided by FB (between January 2000 and April 2008) performed by our group were included. A multivariate analysis of the factors that could influence in the outcome was conducted: cause of the DAW, TI pathway, type of endotracheal tube, elective or urgent indication of the procedure, sedation level and experience of the bronchoscopist. 
Results. Tracheal intubation in individuals who are going to undergo surgical interventions accounts for $92.6 \%$ of the total. The most frequent indications of TI by FB were: limitation of neck movement (60 cases), airway stenosis (24), increase of soft tissues (13), narrow oral aperture (9), airway compression (6), and vocal cord paralysis (6). In $10(8.2 \%)$ cases, TI by FB was not possible. The variables that best predicted IT failure in the multivariate analysis were profound sedation/ general anesthesia $(\mathrm{OR}=12.2 ; 95 \% \mathrm{Cl}, 1.8-84 ; \mathrm{p}=$ 0.01 ) and limited experience of the bronchoscopist (OR = 25.3; 95\% Cl, 3.5-181.8; $p=0.001$ ).

Conclusions. TI guided by FB performed by bronchoscopist is successful in more than $90 \%$ of the cases with DAW. The skill and experience of the bronchoscopist is one of the primary determining factors of success of the procedure. Profound sedation may condition TI guided by FB failure.

KEY WORDS: Bronchoscopy. Difficult airway. Tracheal intubation.

\section{INTRODUCCIÓN}

Uno de los progresos más importantes en el abordaje de la vía aérea difícil (VAD) ha sido la intubación traqueal (IT) guiada por fibrobroncoscopio $(\mathrm{FB})^{1}$. Se considera una de las técnicas de elección en situaciones en que la laringoscopia es peligrosa ${ }^{2}$, inadecuada o imposible, ya que permite practicar, bajo visión directa, una rápida y atraumática intubación, incluso en los casos con importantes alteraciones anatómicas $^{3,4}$. La incidencia de IT difícil varía enormemente en la literatura, que oscila entre el $0,5 \mathrm{y}$ el $13,6 \%{ }^{5,6}$. Cuando esto ocurre de una forma inesperada, las complicaciones son frecuentes e incluso la vida del paciente puede verse en peligro. A pesar de que la fibrobroncoscopia se ha convertido en una técnica de referencia en los casos de IT difícil, hay importantes limitaciones que condicionan el uso sistemático de esta técnica. Muchos servicios de anestesia no disponen de FB y requieren de la ayuda de otros especialistas para realizar la IT con FB. También es frecuente que, a pesar de disponer de FB, sean pocos los miembros de un servicio de anestesia o intensivos que estén familiarizados con este instrumento. Se ha descrito que con esta técnica el manejo de la VAD es exitoso en el $87-100 \% \%^{7-9}$. Aunque en las guías y manuales de IT se enumeran las posibles causas de fallo para la IT guiada por $\mathrm{FB}^{4,10}$, hay pocos trabajos publicados que analicen la incidencia y las causas del resultado de la IT guiada por $\mathrm{FB}^{9}$ y en ninguno de ellos se ha realizado un análisis estadístico multivariable de los factores que podrían influir en el resultado de esta técnica.

El objetivo del presente estudio es analizar los factores que podrían predecir el fallo de la IT con FB.

\section{MATERIAL Y MÉTODOS}

Estudio observacional retrospectivo, con componente analítico, en el que se incluyeron las últimas
122 intubaciones consecutivas guiadas por FB realizadas en pacientes con VAD, ingresados en el servicio de cuidados intensivos o reanimación de nuestro hospital por insuficiencia respiratoria aguda o bien que se someterían a una intervención con anestesia general, efectuadas por miembros de nuestro servicio. Los procedimientos incluidos en el estudio se realizaron entre enero de 2000 y abril de 2008. La fuente de datos fue el registro de la unidad de broncoscopias de nuestro centro.

A partir de la revisión de la historia clínica del paciente, el registro de actividades quirúrgicas, el informe de la consulta preanestésica y el informe médico de la fibrobroncoscopia, se recopilaron las siguientes variables: la edad y el sexo de los pacientes, la causa por la que se indicó la IT guiada por FB, la especialidad para la que se realizó esta intervención, si la indicación del procedimiento fue urgente o programada, el grado de sedación del paciente, la vía de intubación, el tipo de tubo endotraqueal utilizado y el endoscopista que realizó la fibrobroncoscopia.

Para el análisis estadístico de los factores que podrían predecir el fallo de la intubación, las causas de una VAD se dividieron en 2 grupos: de causa obstructiva, todas aquellas que condicionaban de manera directa o indirecta una disminución del diámetro de la vía aérea, y de causa no obstructiva, todas las alteraciones anatómicas o situaciones patológicas que dificultaban la movilidad del cuello o la apertura de la boca.

Según el grado de sedación se definieron 2 situaciones: respiración espontánea para los pacientes sometidos a la IT sin sedación o con sedación consciente superficial y respiración no espontánea, para los pacientes con sedación profunda en los que se evidenciaron mínimas incursiones respiratorias espontáneas o aquellos con anestesia general.

Se consideró, de forma arbitraria, broncoscopista con experiencia aquel con amplia formación teórica y práctica habitual en técnicas endoscópicas, que realizaba, al menos, 100 fibrobroncoscopias y 5 IT guiadas por broncoscopio anuales, y broncoscopista con escasa experiencia, el facultativo que sólo había realizado técnicas endoscópicas durante su período de formación y luego de forma ocasional.

Se utilizaron varios modelos de FB de la casa Olympus (Tokio, Japón), con un diámetro externo de $6 \mathrm{~mm}$ para las intubaciones en que se emplearon tubos endotraqueales mayores de $7 \mathrm{~mm}$, y un FB pediátrico de $3 \mathrm{~mm}$ de diámetro para las IT con tubos endotraqueales de 4 a $7 \mathrm{~mm}$ de diámetro.

La elección de la vía de intubación (nasal u oral) fue realizada por el anestesista o intensivista responsable; habitualmente se prefirió la intubación orotraqueal y se dejó la vía nasal sólo para cuando la oral no era posible por alteraciones anatómicas o microstomía o si fallaba un intento previo por vía oral. No se emplearon instrumentos especiales de ayuda a la intubación, con la excepción de una cánula oral para evitar el daño del FB en los casos de intubación orotraqueal.

La técnica habitual que se empleó fue la siguiente: antes de la IT se procedió a la anestesia tópica de 
orofaringe y fosas nasales (en los casos de IT por vía nasal) con lidocaína al 2 o el 4\%. La anestesia laringotraqueal se aplicó instilando lidocaína al 2\% sobre las cuerdas vocales a través del canal del FB o bien mediante punción de la membrana cricotiroidea. La preparación y la técnica variaron en algunos casos en que el procedimiento fue urgente y en función del grado de sedación del paciente, es decir, en los casos en que, por la situación de insuficiencia respiratoria del paciente, no era posible realizar adecuadamente todos los pasos de preparación y anestesia.

El tubo endotraqueal se pasaba bien lubricado a través del FB. Cuando el FB había pasado las cuerdas vocales y se encontraba en la tráquea o el bronquio principal, se deslizabael tuboendotraqueal. Finalmente, al tiempo que se retiraba el FB se comprobaba la correcta colocación del tubo, que quedaba $2-3 \mathrm{~cm}$ por encima de la carina traqueal.

\section{Análisis estadístico}

Los resultados se expresaron como porcentajes y frecuencias absolutas para las variables cualitativas y como media e intervalo para las numéricas. La comparación de las variables discretas se llevó a cabo mediante la prueba de la $\chi^{2}$ o el test exacto de Fisher. Se consideró estadísticamente significativa una $\mathrm{p}<0,05$. Todos los factores que, en el análisis univariable, se relacionaron con el fallo de la IT guiada por FB con una $\mathrm{p}<0,1$ (para obtener así un mejor ajuste de posibles variables de confusión) se analizaron de forma multivariable mediante un modelo de regresión logística paso a paso hacia atrás, calculándose las odds ratio (OR) y los intervalos de confianza (IC) del $95 \%$. Los análisis se realizaron con el programa Statistical Package for Social Sciences versión 9.0 (SPSS, Chicago, Estados Unidos).

\section{RESULTADOS}

Se incluyeron las últimas 122 IT consecutivas guiadas por FB efectuadas por nuestro grupo. De ellas, 82 fueron en varones y 39 en mujeres. La media de edad de los pacientes fue 58,5 (intervalo, 1-82) años. Las IT en individuos que se someterían a intervenciones quirúrgicas representaron el 92,6\% del total; fundamentalmente, fueron neuroquirúrgicas (37 casos), de cirugía general (21), otorrinolaríngeas (18) o traumatológicas (13). En 9 casos, se solicitó la IT mediante FB por un cuadro de insuficiencia respiratoria aguda en relación con diversas enfermedades médicas y VAD y, en ocasiones, tras el fallo de intentos previos de IT mediante laringoscopia directa.

Los facultativos responsables de cada paciente consideraron la VAD por el hallazgo de una o varias de las causas que se mencionan en la tabla 1. Las enfermedades más frecuentes que presentaban los pacientes en los que se utilizó la IT con FB se muestran en la tabla 2. En el 37,7\% de los casos había antecedentes de IT difícil. Según la clasificación de Mallampati, el 7,4\% se consideró de clase I; el 20,5\%, de clase II; el 29,5\%, de clase III, y el $31 \%$, de clase IV.

En $10(8,2 \%)$ casos no fue posible llevar a cabo la IT. En 3 casos había obstrucción completa de la vía aérea, uno por carcinoma de laringe y en 2 casos por edema de glotis secundario a shock anafiláctico, por lo que no había espacio suficiente para el paso del FB ni de un tubo de diámetro mínimo para ventilar al paciente y se realizó una traqueotomía de urgencia. En otro caso, al verificar la correcta colocación del tubo endotraqueal, el paciente fue extubado accidentalmente. En 3 casos no se llegó a visualizar las cuerdas vocales y en otros 3 , aunque se introdujo el FB en la tráquea, no fue posible hacer progresar el tubo endotraqueal.

TABLA 1. Causas de vía aérea difícil

\begin{tabular}{|l|c|}
\hline Causas & Pacientes (n) \\
\hline Laringoscopia difícil & 60 \\
Limitación del movimiento del cuello & 14 \\
Apertura oral limitada & 10 \\
Cuello grueso o corto & 13 \\
Aumento de tejidos blandos & 2 \\
Micrognatia & 7 \\
Otras* & \\
Dificultad para insertar el tubo endotraqueal & 24 \\
Estenosis de vía aérea & 6 \\
Parálisis de cuerdas vocales & 6 \\
Compresión traqueal & 4 \\
\hline Otras* & \\
\hline
\end{tabular}

*Incluye macroglosia, alteraciones o distorsiones anatómicas.

Nota: En algún caso se combinaron varias causas de vía aérea difícil.

TABLA 2. Enfermedades relacionadas con una intubación traqueal difícil

\begin{tabular}{|c|c|}
\hline Enfermedades & Pacientes (n) \\
\hline Traumatismos/cirugía cervical & 34 \\
\hline Carcinoma de laringe & 13 \\
\hline \multicolumn{2}{|l|}{ Rigidez cervical } \\
\hline Artritis reumatoide & 9 \\
\hline Espondilitis anquilosante & 6 \\
\hline Espondiloartrosis & 8 \\
\hline Masa/tumoración mediastínica & 5 \\
\hline Obesidad mórbida/SAHS & 9 \\
\hline Bocio & 2 \\
\hline Otras* & 15 \\
\hline \multicolumn{2}{|c|}{$\begin{array}{l}\text { SAHS: síndrome de apnea-hipopnea del sueño. } \\
\text { *Incluye cambios posquirúrgicos o por radioterapia, deformidades congénitas, tras- } \\
\text { tornos neuroendocrinos. } \\
\text { Nota: En algún caso no se evidenció una enfermedad específica y en otros se com- } \\
\text { binaban más de una. }\end{array}$} \\
\hline
\end{tabular}


En tres de los casos con fallo de la IT guiada por FB no había una causa obstructiva de VAD. Dos de ellos presentaban clase III en la clasificación de Mallampati y uno, clase II. En los 3 pacientes el procedimiento fue urgente.

En el análisis univariable los factores que predijeron el fallo de una IT guiada por FB fueron que el paciente no presentara respiración espontánea durante el procedimiento, que el facultativo que realizó la IT fuese un broncoscopista con escasa experiencia y que la indicación fuese urgente (tabla 3). Entre los pacientes con sedación profunda, el 4,1\% (5 pacientes) recibió relajación muscular. Un tercio de las IT urgentes fueron realizadas por broncoscopistas poco experimentados frente a un $12 \%$ de las programadas $(\mathrm{p}=0,01)$.

\begin{tabular}{|c|c|c|}
\hline Factores & Fallo de la intubación & $\mathrm{p}$ \\
\hline Enfermedad & & 0,15 \\
\hline Obstructiva & $6 / 49(12,2 \%)$ & \\
\hline No obstructiva & $4 / 73(5,5 \%)$ & \\
\hline Tipo de tubo & & 0,55 \\
\hline Flexometálico & $2 / 49(4,1 \%)$ & \\
\hline Otros & $2 / 35(5,7 \%)$ & \\
\hline Vía de intubación & & 0,7 \\
\hline Oral & $9 / 110(8,2 \%)$ & \\
\hline Nasal & $1 / 8(12,5 \%)$ & \\
\hline Broncoscopista & & $<0,001$ \\
\hline Con escasa experiencia & $8 / 21(38,1 \%)$ & \\
\hline Con experiencia & $2 / 101(2 \%)$ & \\
\hline Petición & & 0,002 \\
\hline Urgente & $7 / 30(23,3 \%)$ & \\
\hline Programada & $3 / 92(3,3 \%)$ & \\
\hline Grado de sedación & & 0,001 \\
\hline $\begin{array}{l}\text { Respiración } \\
\text { no espontánea }\end{array}$ & $6 / 20(30 \%)$ & \\
\hline Respiración espontánea & $3 / 97 *(3,1 \%)$ & \\
\hline
\end{tabular}

*5 $(4,1 \%)$ casos con relajación muscular.

TABLA 4. Regresión logística de los factores que predicen el fallo de la intubación traqueal guiada por fibrobroncoscopio

\begin{tabular}{l|c|c|c|}
\hline Factores & OR & IC del 95\% & $\mathrm{p}$ \\
\hline Petición urgente & 3,3 & $0,5-20,3$ & 0,19 \\
Respiración no espontánea & 12,2 & $1,8-84$ & 0,01 \\
$\begin{array}{l}\text { Broncoscopista con escasa } \\
\quad \text { experiencia }\end{array}$ & 25,3 & $3,5-181,8$ & 0,001 \\
\hline
\end{tabular}

En la tabla 4 se muestran los resultados del modelo de regresión logística aplicada. Los factores relacionados de forma independiente con el fallo de la IT con FB fueron la falta de experiencia del broncoscopista y que la IT se realizara en pacientes con sedación profunda o anestesia general.

\section{DISCUSIÓN}

La IT asistida con FB ha sido uno de los progresos más importantes en el abordaje de la $\mathrm{VAD}^{3}$. Se ha consolidado como una de las técnicas más seguras y resolutivas en el paciente con $\mathrm{VAD}^{3,4}$. Presenta la enorme ventaja de la visualización directa y es una técnica poco traumática y bien tolerada por el paciente $\mathrm{e}^{4,7,11}$.

El resultado comunicado de la IT guiada por FB ha sido variable, y oscila en el $87-100 \%$ de éxitos en casos de $\mathrm{VAD}^{7-9}$. En algunas guías clínicas se describen, como posibles causas de fallo para la IT guiada por $\mathrm{FB}^{4,10}$, la reducción de la luz laringotraqueal, la sedación profunda o la inexperiencia del broncoscopista $^{12}$. Sin embargo, la influencia que estos y otros factores pueden tener en predecir el resultado de la intubación no se ha analizado específicamente y sólo opiniones de expertos y algún estudio avalan estas aseveraciones $^{9,12}$.

En nuestra serie, en 10 casos no fue posible la IT guiada por FB. Tras el análisis multivariable, los factores que mejor predijeron el fallo de este procedimiento fueron la experiencia del broncoscopista y el grado de sedación del paciente.

Una de las posibles causas de fallo de la IT guiada por FB referidas era la reducción de la luz laringotraqueal por edema, tumores o estenosis ${ }^{4,10}$. En nuestro caso este factor no se relacionó con el resultado de la IT. Se ha descrito que la presencia de sangre y secreciones puede condicionar el resultado de la $\mathrm{IT}^{10}$, factores no recogidos en nuestro trabajo y que podrían haber influido. Delaney et $\mathrm{al}^{13}$ describieron su experiencia con 60 IT guiadas por FB. A pesar de excluir a los pacientes con obstrucción completa de la vía aérea, el fallo fue del $13 \%{ }^{13}$. El resultado de la IT guiada por FB probablemente esté condicionado por otros factores más importantes. En nuestra serie encontramos que en 3 casos en los que falló la IT guiada por FB no había una causa obstructiva; sin embargo, eran procedimientos realizados de forma urgente, que sí es un factor que se relaciona con mayor riesgo de fallo.

También se ha comunicado que el tipo de tubo endotraqueal podría influir en el resultado de la IT guiada por FB. Se ha observado una mayor tasa de éxitos con tubos endotraqueales flexibles que con tubos endotraqueales convencionales rígidos ${ }^{14}$. En un estudio prospectivo, 19 de 20 IT con tubos flexometálicos fueron exitosas al primer intento, frente a 7 de 20 intubaciones con tubos habituales ${ }^{14}$. Nosotros no hemos encontrado diferencias significativas al comparar el resultado de la IT con FB y el tipo de tubo. Al tratarse de un estudio retrospectivo, no disponemos de datos sobre este aspecto en todos los casos, lo que 
podría haber influido en el análisis. Por otro lado, es posible que en la selección del tipo de tubo influya la causa de la VAD, lo que podría complicar aún más la interpretación de los resultados.

Aunque la IT con FB por vía oral puede ser una técnica más difícil de realizar que la intubación por vía nasal ${ }^{4}$, una de las mayores ventajas es el menor riesgo de hemorragia, complicación que puede dificultar el procedimiento en algún caso. Nosotros no encontramos diferencias significativas en el resultado de la IT al comparar ambas técnicas, si bien la vía nasal, debido a la elección de los anestesistas o intensivistas de nuestro centro, en ocasiones condicionada por la posibilidad de una IT prolongada y el riesgo de afección sinusal que ello conlleva, se consideró siempre como una alternativa a la vía oral.

La efectividad de este procedimiento en un paciente con VAD requiere de experiencia. Un trabajo previo demostró que, tras un programa específico de formación en IT guiada por FB, se alcanzaba un éxito de casi el $100 \%{ }^{15}$. La mayoría de los estudios han sido realizados por médicos con amplia experiencia en técnicas endoscópicas y en el manejo de la $\mathrm{VAD}^{7-9,13}$. En nuestra serie, observamos un mayor porcentaje de fallos cuando la IT la realizó un broncoscopista con poca experiencia.

Muchos de los autores consideran la IT con FB de elección para el manejo de la VAD en el paciente con respiración espontánea ${ }^{8,16}$. En las guías de la $\mathrm{ASA}^{3}$, los expertos acentúan la importancia de esta técnica en estos casos, pero son menos entusiastas en los pacientes anestesiados. La IT en pacientes con anestesia general tiene 2 importantes desventajas: la limitación en el tiempo impuesta por la urgencia de la IT a un paciente en apnea y la pérdida de tono y control de la musculatura faringolaríngea, que puede producir un colapso y convertirse en un obstáculo insuperable. Ovasappian et $\mathrm{al}^{9}$, en una serie de 409 IT, solamente describen un 1,2\% de fallos. En su serie, únicamente se realizó IT con anestesia general a 5 pacientes. En nuestro estudio la sedación profunda/anestesia general demostró ser un factor independiente del fallo de la IT con FB.

Para asegurar un buen resultado, la IT con FB debe practicarse de forma periódica y no reservarse exclusivamente para situaciones de urgencia. En algunos de los trabajos publicados sólo se incluyeron procedimientos programados ${ }^{9,16}$. Hemos observado diferencias en el resultado de la IT cuando el procedimiento se realiza de forma programada o cuando éste es urgente, aunque este factor no alcanzó significación estadística tras ajustarlo según el grado de sedación y la experiencia del especialista en la técnica. En nuestra serie, la cuarta parte de los procedimientos se realizaron de forma urgente, con el paciente menos preparado e intentos previos de IT por laringoscopia directa infructuosos. Esto puede aumentar el edema, el sangrado y las secreciones, todos ellos causas potenciales de fallo. Otro aspecto importante es que los broncoscopistas menos experimentados realizaron significativamente un mayor número de broncoscopias urgentes que los experimentados, ya que cuando la IT por FB es programada la realizan habitualmente los endoscopistas habituales del centro y en el caso de las urgentes, al realizarse muchas durante horario de atención continuada, frecuentemente son realizadas por neumólogos con menos experiencia en el manejo de la vía aérea.

En la literatura médica podemos encontrar algunas series que describen y aportan su experiencia en este procedimiento con una casuística mucho más amplia $^{7,8}$. El escaso número de procedimientos realizados en un período de estudio tan amplio, en relación con el número de intervenciones quirúrgicas llevadas a cabo en ese tiempo, nos hace pensar que los casos analizados fueron muy seleccionados y, probablemente, con una franca VAD. Esto podría haber influido también en el resultado general.

Hay instrumentos de ayuda a la intubación con FB que facilitan su inserción y/o el mantenimiento de la ventilación del paciente durante el procedimiento ${ }^{17}$. Además, para resolver los casos de VAD, en los últimos años se han diseñado otros sistemas, como la mascarilla laríngea de intubación, Fastrach ${ }^{\circledR}$ o estiletes con luz, entre otros, y nuevos dispositivos como el laringoscopio de Bullard ${ }^{18}$. Sin embargo, este estudio únicamente pretende valorar la capacidad de la fibrobroncoscopia simple en el manejo de la VAD. Los dispositivos de ayuda para la IT pueden ser especialmente útiles en los casos que se cumplan los factores predictores de fallo encontrados en el presente estudio. Creemos que los resultados obtenidos pueden ser de interés para cualquier profesional con responsabilidad en el manejo de la vía aérea, como intensivistas, anestesistas, neumólogos y, en algunos casos, otros especialistas de las áreas quirúrgicas. La IT con $\mathrm{FB}$ con el enfermo despierto es uno de los métodos de control de la vía aérea recomendado en los casos de dificultad prevista de intubación que, con formación y entrenamiento apropiados, todo profesional implicado en el manejo de la vía aérea puede realizar.

A pesar de las limitaciones del trabajo ya referidas, el tamaño muestral escaso de nuestra serie, que es la causa de los amplios intervalos de confianza en el estudio multivariable, y las habituales de todo estudio retrospectivo, creemos que la IT guiada por FB, realizada por broncoscopistas, es exitosa en un elevado porcentaje de casos. Sin embargo, el resultado de esta técnica derivará de dos factores fundamentales. En primer lugar, la habilidad y la experiencia del facultativo en el procedimiento son factores determinantes en el resultado. La IT guiada por FB deben realizarla especialistas con experiencia y amplia formación en técnicas endoscópicas. En segundo lugar, es preferible que el procedimiento se realice con el paciente despierto y respiración espontánea. La sedación profunda o la anestesia general podrían condicionar el fallo de la intubación.

\section{AGRADECIMIENTOS}

Estudio realizado gracias al programa de intensificación de la actividad investigadora en el SNS (FIS-ISCIII). 


\section{BIBLIOGRAFÍA}

1. Murphy P. A fibre-optic endoscope used for nasal intubation. Anaesthesia. 1967;22:489-91.

2. Miñambres E, Burón J, González-Castro A, RodríguezBorregán JC, Mons R, López-Espadas F. Rotura traqueal tras intubación urgente. Med Intensiva. 2005;29:393-5.

3. Caplan RA, Benumof JL, Berry FA, Blitt CD, Connis RT, Guidry OF, et al. Practice guidelines for management of the difficult airway. An updated report by the American Society of Anesthesiologists Task Force on management of the difficult airway. Anesthesiology. 2003;98:1269-77.

4. Navío Martín MP, Cadenas Álvarez AM, Domínguez Reboiras S. Broncoscopia terapéutica en el manejo de la vía aérea. En: DíazAgero Álvarez P, Flandes Aldeyturriaga J, editores. Broncoscopia diagnóstica y terapéutica. Monografías Neumomadrid. Madrid: Ergón; 2007. p. 99-116.

5. Bellhouse CP. An angulated laryngoscope for routine and difficult tracheal intubation. Anesthesiology. 1988;69:126-9.

6. Wilson ME, Spielgelhalter D, Robertson JA, Lesser P. Predicting difficult intubation. Br J Anaesth. 1988;61:211-6.

7. Cámara Angulo F, Domínguez Reboiras S, Martín Burcio S, Pacheco Galván A. Intubación traqueal mediante broncofibroscopia (experiencia de un servicio hospitalario). Arch Bronconeumol. 1996; 32:379-83.

8. Martínez Abad Y, Puente Maeztu L, Sánchez Muñoz G, Pedraza Serrano F. Intubaciones mediante fibrobroncoscopio. Rev Patol Respir. 2006;9:175-8.

9. Ovassapian A, Yelich SJ, Dykes MHM, Brunner EE. Fiberoptic nasotracheal intubation. Incidence and causes of failure. Anesth Analg. 1983;62:692-5.
10. Ovassapian A. The flexible bronchoscope. A tool for anesthesiologists. Clinics in Chest Med. 2001;22:281-9.

11. Muñoz-Martínez T, Pardo-Rey C, Silva-Obregón JA. Sedación en procedimientos y situaciones especiales. Med Intensiva. 2008;32:s107-14.

12. Koerner IP, Brambrink AM. Fiberoptic techniques. Best Pract Res Clin Anaesthesiol. 2005;19:611-21.

13. Delaney KA, Hessler R. Emergency flexible fiberoptic nasotracheal intubation: a report of 60 cases. Ann Emerg Med. 1988; $17: 919-26$

14. Brull JS, Wiklund R, Ferris C, Cornnelly NR, Ehrenwerth J, Silverman DG. Facilitation of fiberoptic orotracheal intubation with a flexible tracheal tube. Anesth Analg. 1994; 78:746-8

15. Ovassapian A, Dykes MHM. A training programme for fibreoptic nasotracheal intubation. Use of model and live patients. Anaesthesia. 1983;38:795-8

16. Casals P, Hernando D, Martínez D, Mulas D, Cochs J Viguera M, et al. Utilización del fibrobroncoscopio para la intubación en cirugía maxilofacial. Rev Española Anest Rean. 1987;34:62-6.

17. Greenland KB, Ha ID, Irwin MG. Comparison of the Berman Intubating airway and the Williams Airway Intubator for fibreoptic orotracheal intubation in anaesthetised patients. Anaesthesia. 2006;61:678-84.

18. Henderson JJ, Popat MT, Latto IP, Pearce AC. Difficult airway society guidelines for management of the unanticipated difficult intubation. Anaesthesia. 2004;59:675-94. 